\title{
Accuracy of measurements of basic parameters of observation thermal cameras
}

\author{
by J. Barela, M. Kastek K. Firmanty and P. Trzaskawka
}

Miliraty University of Technology, 00-908, S. Kaliskiego Str., Warsaw, Poland, jaroslaw.barela@wat.edu.pl

\begin{abstract}
Measurements of performance parameters of observation thermal cameras are usually conducted to check the applied design concept and the operation of implemented image-enhancing algorithms or to verify the manufacturer's claim regarding the figures of merit. In both cases there are several important conditions to be met in order to obtain reliable measurement results. One must have a measurement stand providing measurement uncertainty figures several times lower than the magnitude of measured parameter. There can be no influence of environmental conditions and the personnel conducting the test must precisely know the applicable procedures and should possess considerable experience in the measurements of thermal imaging devices. The paper presents the measurement equipment used to test the infrared imaging devices as well as measurement data analysis regarding basic camera parameters. Accuracy assessment were conducted on the basis of measurement methods and procedures implemented in Accredited Testing Laboratory of Institute of Optoelectronics, Military University of Technology.
\end{abstract}

\section{Introduction}

There are dozens of performance parameters of observation thermal cameras that can be measured. They can be divided into several groups on the basis of measurement method or information that they carry [[1][3]. The progress in infrared technology area renders some of those parameters unusable or obsolete, whereas some new, emerging ones gain importance as they are better suited to describe modern thermal cameras [4]. There is, however, a group of performance parameters commonly used as figures of merit for years and still being extremely useful in characterizing the performances of IR cameras of today. Those are: noise equivalent temperature difference (or thermal resolution) NETD, minimum resolvable temperature difference MRTD and modulation transfer function (MTF).

Performance parameters of thermal cameras are measured according to mandatory standards [5][7] , published procedures [4] [9][13] of factory and laboratory standards [14][15]. It is important to apply current measurement procedures, which is particularly evident while using NATO standards. They are still mandatory, valid ones, but much has changed in the infrared and measurement technology area since their publication date.

Measurement test stands, their construction and specifications, are widely described in literature [16][19]. There are several dozens of manufacturers of such equipment on the market, offering fairly similar designs. Main differences are in the exact parameters of the applied IR source (blackbody), collimator, measurement transducers and the capabilities to measure particular set of performance characteristics of a tested IR camera [20][23].

Measurement error assessment consists of the evaluation of the influence of particular factors on the final measurement result, which must include errors introduced by measurement equipment itself as well as inherent errors of the applied measurement method. Analysis was performed according to current standards and recommendations issued by national certification body - Polish Centre for Accreditation [24][28].

\section{Description of the measurement stand}

Test stand used to determine the performance parameters of observation thermal cameras consists of: - infrared source (blackbody),

- collimator,

- rotating wheel with a set of test patterns,

- data acquisition (DAQ) boards

- computer with test software.

Operation of the test stand is as follows: Rotating wheel with test patterns is located at a focal plane of an infrared collimator. The wheel itself is surrounded by a metal cover and the temperature of those two elements is the same. The cover has the blackbody attached to it and an opening at the same height as the test pattern. As a result the specific temperature distribution at the focal plane of a collimator is obtained - test pattern at blackbody temperature against uniform background at ambient. By controlling the blackbody temperature the thermal contrast between test pattern and ambient can be adjusted, and both positive and negative values can be obtained as it is required during measurements. Observation cameras are meant to view objects at considerable distances, hence the application of an infrared collimator creating the image of a test pattern at infinity. Furthermore the use of a collimator with a set of aperture stops makes it possible to eliminate the influence of external heat sources (like personnel and other objects in the laboratory) on the test results. 
a)

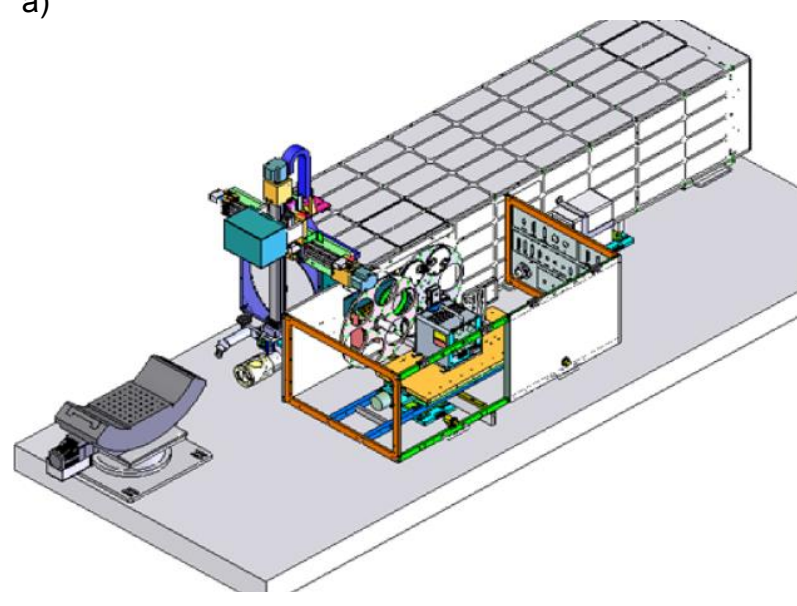

b)

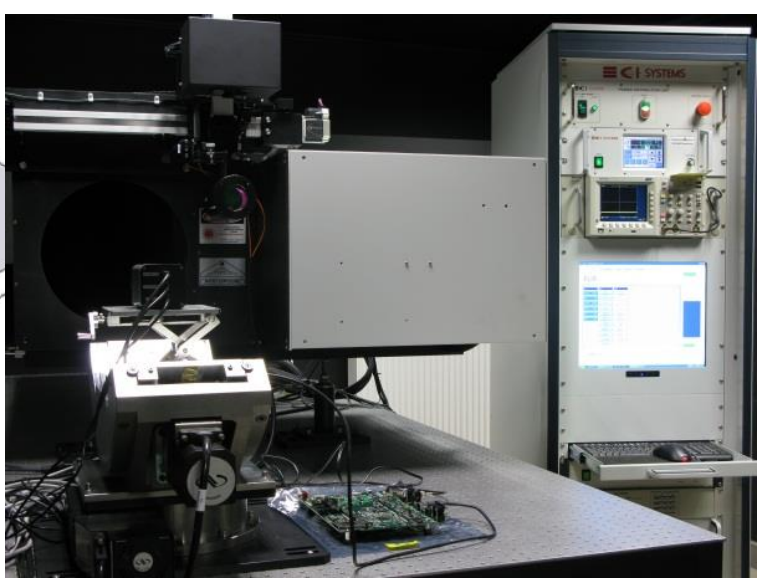

Fig.1. Test stand for the measurements of parameters of observation thermal cameras: a) structure diagram, b) real photo.

All of the analysis and measurement results presented here were obtained on the basis of many years of experience in camera testing at Accredited Testing Laboratory of Institute of Optoelectronics, Military University of Technology. The laboratory is equipped with two measurement stands: MST and METS, both made by $\mathrm{Cl}$ Systems (Fig. 1). Basic specifications of those test stands are given in Tables 1-4. Using these equipment, the following measures for assessing the performance of observation thermal cameras can be evaluated:

- modulation transfer function MTF (using narrow slit method and edge method),

- pre-sample MTF,

- contrast transfer function CTF,

- signal-to-noise ratio SNR in output image,

- minimum resolvable temperature difference MRTD,

- minimum detectable temperature difference MDTD,

- minimum temperature difference perceived MTDP,

- noise equivalent temperature difference NETD,

- $3 D$ noise and $1 / f$ noise,

- signal transfer function SiTF,

- noise power spectral density NPSD,

- inhomogeneity equivalent temperature difference IETD,

- average modulation at optimum phase $A M O P$,

- observational uniformity,

- field of view.

Table 1. Blackbody SR800R-4D-HE specifications

\begin{tabular}{|l|l|}
\hline Parameter & Value \\
\hline Temperature control & thermoelectric \\
\hline Aperture diameter & $4^{\prime} \times 4^{\prime}$ \\
\hline Differential temperature & $-30^{\circ} \mathrm{C} \div 100^{\circ} \mathrm{C}$ \\
\hline Emissivity & $0.98 \pm 0.01$ \\
\hline Stability & $\pm 0,002^{\circ} \mathrm{C}$ \\
\hline Uniformity & $\pm 0,01^{\circ} \mathrm{C}$ \\
\hline
\end{tabular}

Table 2. Collimator METS L-12 specifications

\begin{tabular}{|l|c|}
\hline Parameter & Value \\
\hline Aperture & $300 \mathrm{~mm}$ \\
\hline Focal length & $1787 \mathrm{~mm}$ \\
\hline Transmission coefficient $\tau$ & $\geq 0,89$ (VIS) \\
& $\geq 0,98$ (LWIR) \\
& $\geq 0,99$ (MWIR) \\
\hline Resolution & diffraction limited \\
\hline
\end{tabular}


Table 3. Frame graber Solios eA/XA specifications

\begin{tabular}{|l|c|}
\hline \multicolumn{1}{|c|}{ Parameter } & Value \\
\hline A/D converter & $10 \mathrm{bit}$ \\
\hline Input voltages & $0 \div 2,4 \mathrm{~V}$ \\
\hline Bandwidth & $100 \mathrm{MHz}$ \\
\hline Data transfer rate & $130 \mathrm{Mbit} / \mathrm{sec}$. \\
\hline Impedance & $75 \Omega$ \\
\hline Limiting error & $\pm 1 \mathrm{LSB}$ \\
\hline
\end{tabular}

Tab. 4. Frame grabber Solios eV-CL specifications

\begin{tabular}{|l|c|}
\hline \multicolumn{1}{|c|}{ Parameter } & Value \\
\hline Max number of pixels per line & $64 \mathrm{~K}$ \\
\hline Max number of lines per frame & $64 \mathrm{~K}$ \\
\hline Data transfer rate & $660 \mathrm{Mbites} / \mathrm{sec}$. \\
\hline Input signal resolution & 16 -bit (1 input) \\
& 12 -bit (2 input) \\
& 8-bit (3 input-RGB) \\
\hline Input voltages & $-0,7 \div 5,7 \mathrm{~V}$ \\
\hline Limiting error & $\pm 1 \mathrm{LSB}$ \\
\hline
\end{tabular}

\section{NETD measurements.}

Noise equivalent temperature difference NETD determines minimum level of an input signal (temperature difference between target and background) which results in signal-to-noise ratio $S N R=1$ in an output image. This parameter is often called thermal resolution of an infrared camera. NETD value depends on high frequency temporal noise. The noise causes differences in the output values of the very same pixel in two consecutive images. There are several noise components contributing to the high frequency temporal noise: Johnson-Nyquist noise, generationrecombination noise and shot noise of detectors and electronic circuits. NETD value for measurement-class cameras defines the theoretical limit of temperature measurement error, whereas for observation infrared cameras it determines the minimum level of incoming signal the camera is able to detect. .

In order to determine NETD the following voltages must be measured: RMS value of spatial noise voltage $U_{n}$, signal voltage $U_{2}$ generated by a test at temperature $T_{2}$, signal voltage $U_{1}$ resulting from background temperature $T_{1}$. Emissivity of both test and background surfaces should be sufficiently high. Temperature difference between background temperature $T_{1}$ and test temperature $T_{2}$ should be determined from previously measured signal transfer function SiTF. Tested infrared device should be mounted in such a way to obtain the test image at the center of viewed area. NETD value is then calculated from the measured values according to the following formula:

$$
N E T D=\frac{\left(T_{1}-T_{2}\right) \cdot U_{n}}{U_{2}-U_{1}}
$$

where: NETD - high frequency temporal noise, $T_{1}$ - background temperature, $T_{2}$ - test temperature, $U_{n}-\mathrm{RMS}$ value of noise voltage, $U_{1}$ - background signal voltage, $U_{2}$ - test signal voltage.

\subsection{Analysis of NETD measurement results}

Measurement uncertainty analysis is performed by evaluating the measurement process. Two approaches to uncertainty evaluation are defined: type A and type B. Type A uncertainty evaluation is applied for repeatedly measured values of a given quantity using statistical analysis. Type B evaluation utilizes scientific judgment or other information concerning the possible values of the measured quantity, influenced by limited accuracy of measured equipment and human imperfections.

NETD value is determined by measuring the RS value of noise voltage and signal voltages corresponding to two different blackbody temperatures. In the applied method the measurements are repeated 50 times and average values are calculated. During the validation of the measurement procedure it was determined, that main source of measurement uncertainty in this case are the errors introduced by the measurement equipment. Thus, using mathematical description of the measurement process the expanded uncertainty in this case can be calculated as:

$$
U(N E T D)=k \cdot\left(\frac{1}{\sqrt{3}} \cdot \frac{\delta S_{\max }}{S_{n}}+\sqrt{\left(\frac{\delta S_{\max }}{S_{2} \cdot \sqrt{3}}\right)^{2}+\left(\frac{\delta S_{\max }}{S_{1} \cdot \sqrt{3}}\right)^{2}+\left(\frac{\delta T_{2}}{T_{2}}\right)^{2}+\left(\frac{\delta T_{1}}{T_{1}}\right)^{2}}\right)
$$

where: $S_{1}, S_{2}$ - mean values of signal voltages for blackbody temperatures $T_{1}$ and $T_{2}$, respectively, $T_{1}, T_{2}-$ blackbody temperatures set during measurements, $\delta S_{\max },-$ limiting error of DAQ board (manufacturer's specifications), $k$ - coverage factor. 


\subsection{1/qirt.2016.004}

\section{MTF measurements.}

Modulation transfer function MTF describes image distortion introduced, in a case considered here, by a thermal imaging device. It can be defined by a Fourier transform of a point spread function (luminance distribution of a point light source) normalized to unity for a zero spatial frequency (Fig. 2).

MTF describes the relation between the original amplitude of sinusoid exitance distribution of a test pattern and the amplitude observed in the output image, as a function of spatial frequency of a test pattern. MTF function is, in a general case, a two-dimensional one. However, for thermal images, two one-dimensional functions (horizontal and vertical) are usually calculated. Measurement of MTF function is objective, can be automated and it is not a time-consuming venture. Additional benefit of MTF is that it can be calculated for the entire device as well as for its particular functional blocks. This can be useful during the designing of new observation devices.

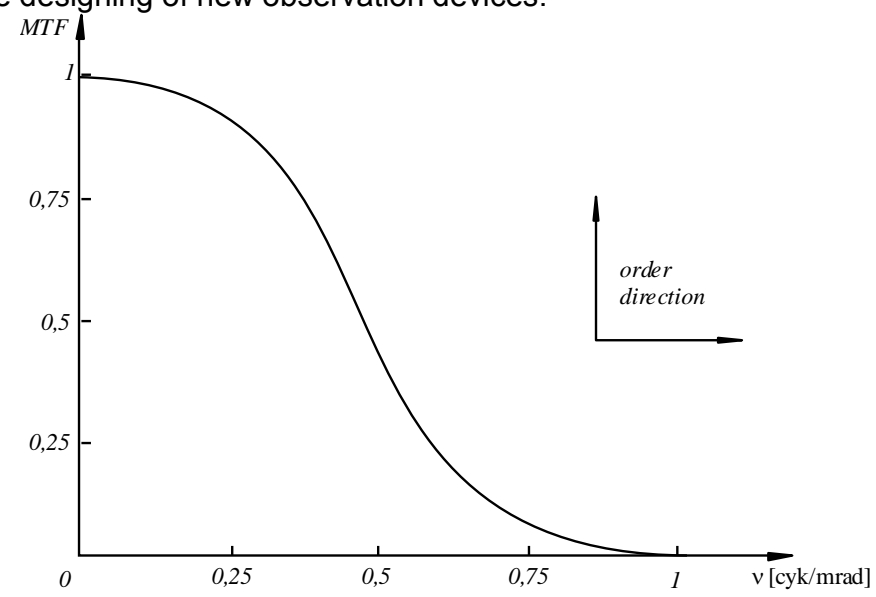

Fig. 2. Sample plot of MTF function

Most common method used to measure MTF function of thermal cameras is edge method. It is a standardized procedure [7] and even though there are some modifications, all of them rely on the same principle (Fig.3). The measurement consists in edge spread function ESF measurements and then calculation of MTF function from it. Edge spread function describes the edge signal distribution as a function of test location on the detector plane (Fig. 4). Edge test should be placed in such a way that its image is parallel to either lines or columns of an array detector.

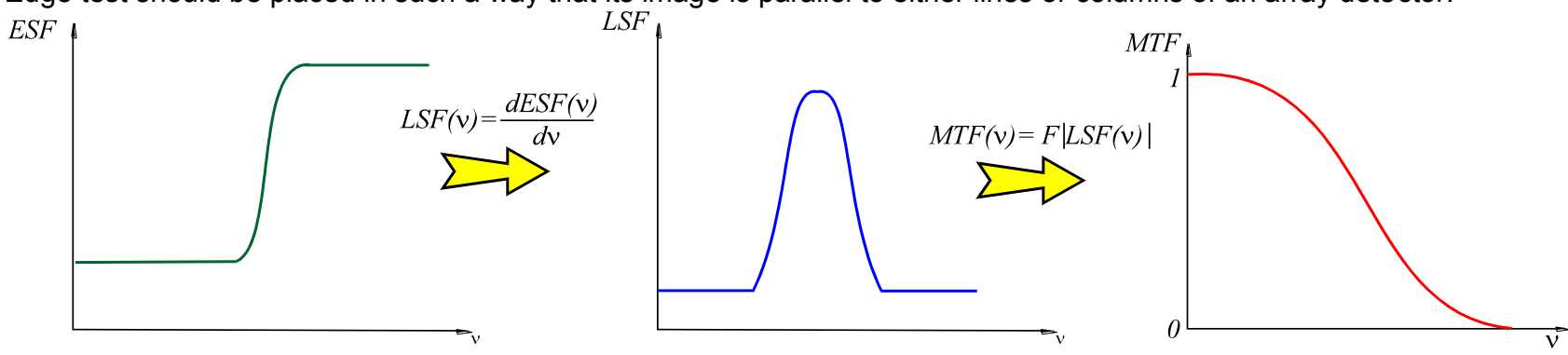

Fig. 3. Determination of MTF function on the basis of measured ESF

The procedure of MTF calculation on the basis of measured ESF function is accomplished in three stages. First the image of an edge test pattern is acquired and an average value of ESF function is calculated. Next line spread function $L S F$ is determined. Average value of $L S F$ function is calculated according to the following relation:

$$
L S F_{\text {ave }}(v)=\frac{d E S F_{\text {ave }}(v)}{d v}
$$

where: $E S F_{\text {ave }}(v)$ ia an average value of edge spread function.

Because the differentiation increases the amount of noise (so-called virtual noise appears) then prior to this operation the measured ESF function is approximated by a differentiable mathematical function which provides the required approximation level. Sufficient accuracy can be obtained by using a sum of three Fermi functions: 
10.21611/qirt.2016.004

$$
F(x)=D+\sum_{i=0}^{2} \frac{a_{i}}{\exp \left[\frac{x-b_{i}}{c_{i}}\right]+1}
$$

where: $D, a_{i}, b_{i}, c_{i}$ are constant values calculated from average signal values before and after edge.

MTF function is then calculated from previously determined LSF using the following formula:

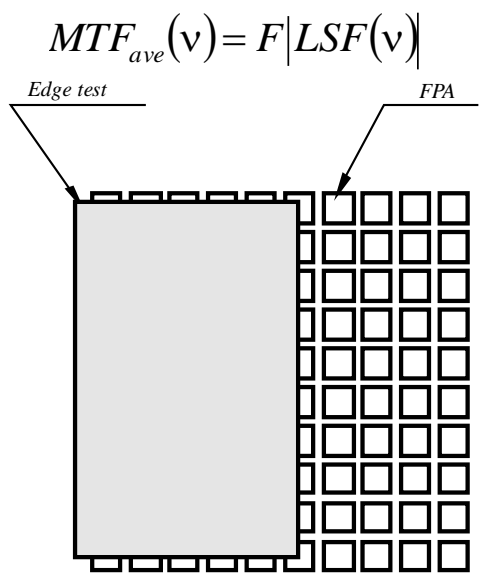

Fig. 4. Edge test image on the detector plane

\subsection{Analysis of MTF measurement results}

The result of MTF measurement is a function, so in the uncertainty analysis only the measurement equipmentrelated errors can be taken into account. The function is naturally measured repeatedly several dozen times during the measurement procedure, resulting in average function value. As a result, type $A$ uncertainty evaluation approach cannot be used. During the measurement procedure the signal values on the test surface are measured. During MTF calculations from ESF function the linear filtration theory is used, thus the camera must operate in its linear range of temperature-to-signal conversion curve, with appropriate signal-to-noise ratio. Temperature difference between test and ambient is determined on the basis of previously measured SiTF function. There are two main error sources during measurements. First, the temperature difference between test and ambient should be constant during the whole measurement procedure. According to blackbody specifications, it is evident that short-term stability of such a device is very high. Thus, this particular factor can be neglected because the voltage measurement errors (second error source) are much greater. The voltage measurement accuracy depends on the performance of applied DAQ board. Using the mathematical model of the measurement process the expanded measurement uncertainty can be determined from the following relation:

$$
U(M T F)=k \cdot\left(\frac{\delta S_{\max }}{S_{n} \cdot \sqrt{3}}\right)
$$

where: $S_{n}$ - signal value on the test surface (calculated from SiTF), $\delta S_{\text {max }}$,- limiting error of DAQ board (manufacturer's specifications), $k$ - coverage factor.

\section{MRTD measurements.}

MRTD characteristics (temperature $\left[\mathrm{K},{ }^{\circ} \mathrm{C}\right]$ versus spatial frequency [lines $\left./ \mathrm{mrad}, \mathrm{mrad}^{-1}\right]$ ) is defined as the relation between minimum temperature difference between a four-bar pattern and background enabling the observer to discern all the pattern bars, and the spatial frequency of the test pattern. Procedure of MRTD determination for thermal imaging devices is described in NATO STANAG no. 4349 [5].

MRTD measurement is a relative one, conducted by human observers. In order to determine MRTD the temperature difference is measured at which the observer can discern all four bars of a test pattern. The observer can adjust gain, screen brightness and use all available controls in the frame of normal camera operating regime and the observation time frame is not limited. 


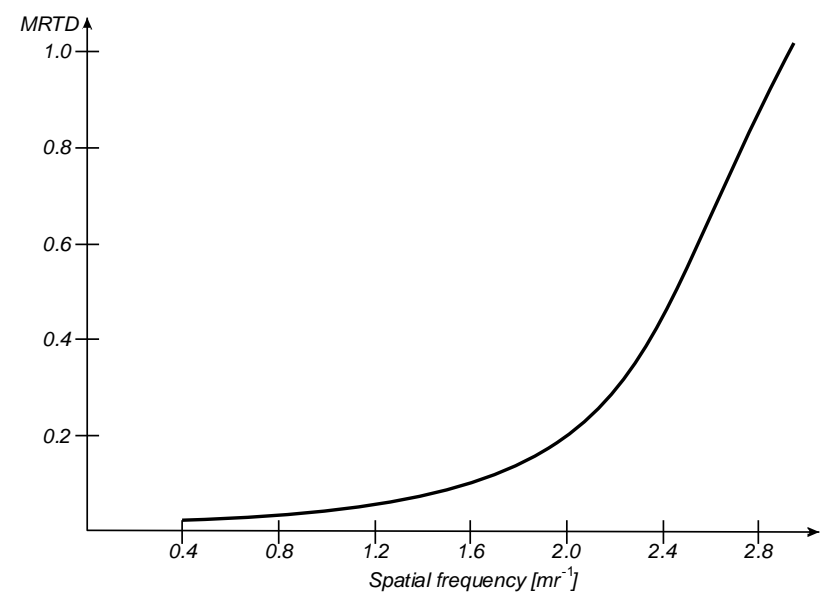

Fig. 5. Sample MRTD characteristic and four-bar test pattern used to measure it

Tests are conducted for both positive (pattern bars hotter than background) and negative (pattern bars colder than background) temperature difference values. In both cases the minimum temperature difference is determined, at which the observer can discern all four bars. Final MRTD values for a particular single observer are calculated from the following equation:

$$
\operatorname{MRTD}(\gamma)=\frac{\Delta T_{+}(\gamma)-\Delta T_{-}(\gamma)}{2}
$$

where: $\triangle T_{+}(\gamma)$ - MRTD values for positive temperature difference at which the observer starts to discern all the bars, $\Delta T$ - $(\gamma)$ - MRTD values for negative temperature difference at which the observer starts to discern all the bars.

It is advised that the tests are performed by three different observers with good eyesight. Final results are given as average values for all observers participating in the testing.

MRTD determination method assumes that final values are obtained as an average value observer's results multiplied by the transmission coefficient of the collimator, according to the following relation:

$$
M R T D=\tau \frac{\left(\frac{\Delta T_{+}^{1}-\Delta T_{-}^{1}}{2}\right)+\left(\frac{\Delta T_{+}^{2}-\Delta T_{-}^{2}}{2}\right)+\left(\frac{\Delta T_{+}^{3}-\Delta T_{-}^{3}}{2}\right)}{3}
$$

where: $\Delta \mathrm{T}_{+}-$positive temperature difference at which the observer starts to discern all the bars, $\Delta \mathrm{T}_{-}-$negative temperature difference at which the observer starts to discern all the bars, $\tau$ - transmission of an infrared collimator. Equation (8) can be rewritten in the following form:

$$
M R T D=\tau\left\{\left(\frac{\Delta T_{+}^{1}+\Delta T_{+}^{2}+\Delta T_{+}^{3}}{6}\right)-\left(\frac{\Delta T_{-}^{1}+\Delta T_{-}^{2}+\Delta T_{-}^{3}}{6}\right)\right\}
$$

\subsection{Analysis of MRTD measurement results.}

In MRTD measurement uncertainty analysis both type A and type B evaluation methods can be applied. Model of the measurement process which includes all relevant error sources can be presented as:

$$
M R T D=\tau\left\{\left(\frac{\sum_{k=1}^{3}\left(\Delta T_{+}^{k}+\Delta T_{s}+\Delta T_{r}\right)}{6}\right)-\left(\frac{\sum_{k=1}^{3}\left(\Delta T_{-}^{k}+\Delta T_{s}+\Delta T_{r}\right)}{6}\right)\right\}
$$

where: $\Delta T_{+}^{k}, \Delta T_{-}^{k}$ - determined values of temperature difference $\Delta \mathrm{T}$ for $k$-th observer, $\Delta T_{s}-$ unknown systematic error of $\Delta \mathrm{T}$ measurement, $\Delta T_{r}$ - random error of $\Delta \mathrm{T}$ measurement caused by limited resolution of a blackbody.

In analysis, systematic error $\Delta T_{s}$ and random error $\Delta T_{r \text {. were }}$ taken into account. Systematic error is a result of limiting error of blackbody temperature stabilization and inaccurate measurements of test temperature. The tests are located on a rotating wheel and the temperature sensor measures in fact wheel temperature, not test temperature. Method of MRTD measurement requires however positive and negative temperature difference values. Then, because systematic error is constant and present on the both sides of temperature differences the relation (10) can be simplified, assuming the following form: 


$$
M R T D=\tau\left\{\left(\frac{\sum_{k=1}^{3}\left(\Delta T_{+}^{k}+\Delta T_{r}\right)}{6}\right)-\left(\frac{\sum_{k=1}^{3}\left(\Delta T_{-}^{k}+\Delta T_{r}\right)}{6}\right)\right\}
$$

The sign in case of random error is irrelevant, thus the relation can be written as:

and finally

$$
M R T D=\tau\left(\frac{\sum_{k=1}^{6}\left(\left|\Delta T^{k}\right|+\Delta T_{r}\right)}{6}\right)
$$

$$
M R T D=\tau\left(\Delta T_{h}+\frac{\Delta T_{r}}{6}\right)
$$

where: $\Delta T_{h}$ - random variable describing the $\Delta T$ temperature difference distribution caused by a limited repeatability of human sight, $\Delta T_{r}$ - random variable describing the distribution of measurement results due to limited thermal resolution of a blackbody.

Finally there are two error sources to be considered: one resulting from limited thermal resolution of a blackbody and second originating from human sight imperfections. MRTD measurements for modern thermal cameras are performed for temperature differences between blackbody and test pattern below $2^{\circ} \mathrm{C}$. According to blackbody specifications it can be stated that standard uncertainty of the random variable $\Delta T_{r}$ is usually below $0,1 \%$. Error caused by limited repeatability of a human sight depends on image noise figures and the test size. It is estimated that for cooled cameras relative standard uncertainty of random variable $\Delta T_{h}$ is usually at around $10 \%$, and $20 \%$ for uncooled cameras. It is then at least hundred times greater than error caused by the measurement stand. As a result the error caused by the measurement equipment can be neglected and relation (13) can be further simplified, yielding the final MRTD value as:

$$
M R T D \cong \tau \Delta T_{h}
$$

Measurement uncertainty for MRTD variable can be calculated from the following relation:

$$
u(M R T D)=\sqrt{\bar{\tau}^{2} \times u^{2}\left(\Delta T_{h}\right)+\Delta \bar{T}_{h} \times u^{2}(\tau)}
$$

where: $u\left(\Delta T_{h}\right)$ - standard uncertainty of $\Delta T_{h}, u(\tau)$ - standard uncertainty of $\tau, \bar{\tau}$ - expected value of $\tau$ variable, $\Delta \bar{T}$ - expected value of $\Delta \mathrm{T}$ variable.

Bearing in mind that uncertainty of $\tau$ variable determination (on the basis of collimator specifications) is negligible comparing to $\Delta T_{h}$ uncertainty, then the extended uncertainty of $M R T D$ measurement can be determined as:

$$
U(M R T D)=k \cdot \frac{\left.\sqrt{\frac{1}{n-1} \sum_{k=1}^{n}\left(\left|\Delta T_{k}\right|-\Delta \bar{T}\right.}\right)^{2}}{\sqrt{n}}
$$

where: $\mathrm{n}$ - number of conducted measurements, $\Delta \bar{T}$ - average value of temperature difference for which the test pattern is discerned, $\left|\Delta T_{k}\right|$ - measured temperature difference for which observer is able to discern the test pattern, $k$ - coverage factor.

\section{Remarks and conclusions.}

Measurement of performance parameters of thermal cameras must be reliable. The test stand itself cannot introduce significant errors affecting the measurement results. It is particularly important to assure stable thermal conditions during the entire measurement procedure. Of course stabilized temperature in the laboratory is the condition required by measurement standards. However even slight air blow from climate control or ventilation may disturb the measurement process and affect the results. This can be observed mainly during MRTD function measurements for cooled IR cameras. Wrong results may also result from human error. Lab personnel must know the measurement procedures and the operating manual of the test stand. Attention should be paid to image enhancing functions which in some cases have to be turned off as they influence the values of the measured parameters.

Analyses of measurement uncertainty presented in this paper were based on test results obtained in Accredited Testing Laboratory of Institute of Optoelectronics, Military University of Technology. The laboratory has been conducting the measurements of performance parameters of thermal imaging devices for many years and its personnel has years of experience in that area. The number of tested cameras to date is well beyond 100 pieces of equipment. The 
measurement equipment itself is a high quality setup with very good specifications. Blackbody IR sources undergo mandatory calibrations on regular basis.

The paper presents the analyses concerning only three performance parameters of observation thermal imaging cameras. Our Laboratory is capable of NETD measurements with expanded uncertainty better than $5 \mathrm{mK}$, and $M T F$ measurements with expanded uncertainty better than $1 \%$ of measured quantity.

In case of MRTD measurements the attainable measurement uncertainty values depend on the camera type and spatial frequency range. For cooled cameras and low spatial frequencies the expanded uncertainty is better than $3 \mathrm{mK}$. For high spatial frequency values the expanded uncertainty is better than $20 \mathrm{mK}$. For uncooled cameras the corresponding expanded uncertainty values are $5 \mathrm{mK}$ and $30 \mathrm{mK}$ for low and high spatial frequencies, respectively. All expanded uncertainty values are given for a coverage factor of two.

\section{REFERENCES}

[1] Gerald C. Holst "Electro-optical imaging system performance", JCD Publishing Company, 2008.

[2] Gerald C. Holst, "Testing and evaluation of infrared imaging systems", JCD Publishing Company, 1993.

[3] K. Chrzanowski "Testing Thermal Imagers. Practical guide“, MUT 2010.

[4] North Atlantic Treaty Organization "Experimental Assessment Parameters and Procedures for Characterization of Advanced Thermal Imagers", 2003.

[5] STANAG 4349,: Measurement of the minimum resolvable temperature difference (MRTD) of thermal cameras, 1995.

[6] STANAG 4347, Definition of nominal static range performance for thermal imaging systems, 1995.

[7] ISO 15529:2010 "Optics and optical instruments: Optical Transfer Function; Measurement of modulation transfer function (MTF) of sampled system".

[8] ISO 9335:1995 „Optics and optical instruments: Optical Transfer Function; Principles and procedures of measurement"

[9] R.G. Driggers, S.J. Pruchnik, Jr., "Laboratory measurement of sampled infrared imaging system performance", Optical Engineering 38 (5), 853-861, (1999).

[10] C. Webb "Approach to 3D noise spectral analysis", Proc. SPIE 2470, 288-299 (1995).

[11] J. Barela, M. Kastek, K. Firmanty, P. Trzaskawka, R. Dulski "Determining detection, recognition and identification ranges of thermal cameras on the basic of laboratory measurements and TTP model“, Proc. SPIE 8355 (2012).

[12] K. Chrzanowski, J. Barela, K. Firmanty, "Testing of electro-optical imaging systems", Design, Analysis, Modeling and Testing XV, G.C. Holst Editor, Proc. of SPIE Vol. 5407 (2004).

[13] R.G. Lyons „Understanding Digital Signal Processing”, WKL Publishing Company (2006).

[14] Modular Electro-optical Test System (METS-L), Operation Manual, (2007).

[15] OIML R 141 "Procedure for calibration and verification of the main characteristic of thermographic instrument", Edition 2008

[16] S.W. Smith „Digital Signal Processing. A Practical Guide for Engineers and Scientists by Steven”, BTC Publishing Company (2003).

[17] R.H.VOLLMERHAUSEN, D.A.REAGO,Jr., R.G.DRIGGERS, Analysis and Evaluation of Sampled imaging Systems, SPIE Press, Bellingham, WA, 2010.

[18] Bareła J.,Firmanty M. Kastek, K.,Polakowski H. ,, Precyzyjne ciało czarne z półprzewodnikowymi modułami termoelektrycznymi." PAK 6/2010.

[19] J. Bareła, M. Kastek, K. Firmanty, H. Polakowski „Weryfikacja parametrów wzorców promieniowania podczerwonego." PAK 10/2011.

[20] www.ci-systems.com.

[21] www.sbir.com.

[22] www.hgh-infrared.com

[23] www.inframet.com.

[24] Wyrażanie niepewności pomiaru: „Przewodnik, Główny Urząd Miar“, Warszawa 1999.

[25] PN-EN ISO/IEC 17025 „Ogólne wymagania dotyczące kompetencji laboratoriów badawczych i wzorcujących”, 2005.

[26] ISO 5725 „Accuracy (trueness and precision) of measurement methods and results.

[27] J. R. Taylor, „Wstęp do analizy błędu pomiarowego”, PWN 2015.

[28] Międzynarodowy Słownik Podstawowych i Ogólnych Terminów Metrologii, GUM,1997. 